\title{
Behaviour and design considerations for continuous flow closed-open-closed liquid microchannels
}

\author{
Jessica Melin, Wouter van der Wijngaart* and Göran Stemme
}

Received 3rd February 2005, Accepted 7th April 2005

First published as an Advance Article on the web 21st April 2005

DOI: $10.1039 / \mathrm{b501781e}$

This paper introduces a method of combining open and closed microchannels in a single component in a novel way which couples the benefits of both open and closed microfluidic systems and introduces interesting on-chip microfluidic behaviour. Fluid behaviour in such a component, based on continuous pressure driven flow and surface tension, is discussed in terms of cross sectional flow behaviour, robustness, flow-pressure performance, and its application to microfluidic interfacing. The closed-open-closed microchannel possesses the versatility of upstream and downstream closed microfluidics along with open fluidic direct access. The device has the advantage of eliminating gas bubbles present upstream when these enter the open channel section. The unique behaviour of this device opens the door to applications including direct liquid sample interfacing without the need for additional and bulky sample tubing.

\section{Introduction}

The majority of microfluidic systems and components are designed using either a fully closed or a fully open approach. Closed microfluidic systems usually comprise a network of microchannels and structures in a substrate which is encapsulated by a lid, creating a contained and controllable environment. Open microfluidics do not have any type of lid, thus leaving the liquid exposed to the ambient environment which may or may not be externally controlled.

Open systems evolved from the microtiter plate, which allowed automation and higher throughput in genomic and proteomic research. Microarrays became the next generation, where molecules are successfully immobilized on certain discrete areas of a plane surface, resulting in arrays of liquid or molecule spots (Agilent, Affymetrix, etc.). Such open systems allow direct access to the liquid sample: however, the risk of contamination can be high unless the ambient environment is controlled. Removal of waste products can be difficult and the risk of evaporation is prevalent. Integrating on-chip liquid control can also be difficult, although Nanogen has a solution using individual electrodes beneath the microarray, which can address individual spots and move the liquids around depending on their electrical characteristics. ${ }^{1} \mathrm{~A}$ variety of other open microfluidic systems exist such as pathways of flowing liquid based on free energies of surfaces (hydrophobic/hydrophilically patterned surfaces), ${ }^{2}$ light driven liquid flow on a photoresponsive surface, ${ }^{3}$ electrowetting (semi-open system), ${ }^{4}$ and nanovials (wells) covered with nonvolatile fluid to reduce evaporation effects. ${ }^{5}$

Closed microfluidic systems range from single meandering microchannels in their simplest form to highly complex multistacked networks of microchannels and integrated functional elements. Closed systems provide a controllable environment and make flow-through systems more easily realizable than do

*wouter@s3.kth.se open systems. Molecules can be immobilized in numerous ways (i.e., by using magnetic beads, $\mu \mathrm{CP}$, mechanical barriers, etc.). Liquid movement can be controlled by integrated methods and components, although many control components are overly complex, bulky and unreliable. Closed systems do require tightly sealed couplers/tubes to interface to the outside world, which can be bulky and occupy expensive on-chip real estate. Trapped gas bubbles are particularly detrimental to closed systems and must be taken into account when designing, since they can block channels, drastically alter the internal geometry of the system and result in unreliable functionality. ${ }^{6}$

Combining open and closed microchannels on a single component in a novel way, as is demonstrated in this paper, couples the benefits of both open and closed microfluidic systems and introduces interesting on-chip microfluidic behaviour. ${ }^{7,8}$ Fluid behaviour in such a component, based on continuous pressure driven flow and surface tension, will be discussed in terms of cross-sectional flow behaviour, robustness, flow-pressure performance, and its application to microfluidic interfacing.

\section{Principle of operation}

The closed-open-closed microchannel carries a continuous flow of pressure driven liquid and consists of a closed inlet channel, closed outlet channel, and a section in between which is open to the environment, see Fig. 1. This flow component can be characterized by the pressure at the inlet (source), $P_{\mathrm{S}}$, pressure at the outlet (drain), $P_{\mathrm{D}}$, and pressure of the open environment, $P_{\text {AIR }}$. By regulating the magnitude and relationship between these pressures, the flow through the microchannel is varied. This is similar to a FET transistor, where changing the source voltage $\left(P_{\mathrm{S}}\right)$, drain voltage $\left(P_{\mathrm{D}}\right)$ and gate voltage $\left(P_{\mathrm{AIR}}\right)$ regulate the device current (liquid flow).

The Laplace equation tells us that the radius of curvature, $r$, for a cylindrical liquid-air interface surface (i.e., similar to the liquid-air surface shape at the open channel section) is 


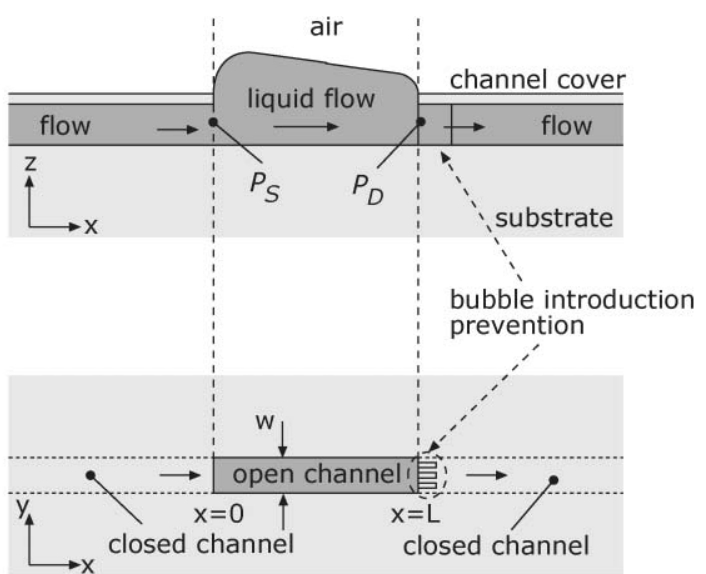

a)

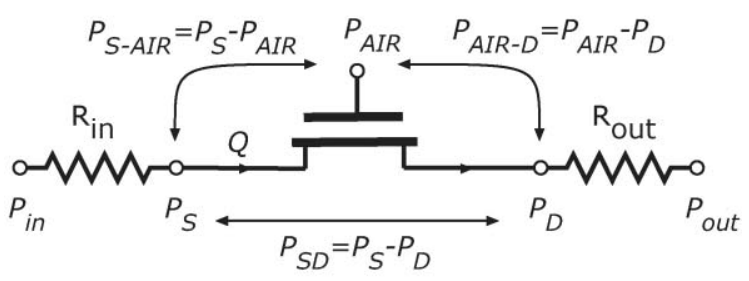

b)

Fig. 1 (a) Cross-sectional lengthwise and bird's eye view of closedopen-closed channel, and (b) transistor analogy.

determined by the pressure difference between the air and liquid in the open channel and can be expressed as

$$
r(x)=\frac{\gamma}{P(x)-P_{\mathrm{AIR}}}
$$

where $\gamma$ is the surface tension of the liquid, $P(x)$ is the liquid pressure at channel position $x$, and $P_{\text {AIR }}$ is the air pressure. Fig. 2 shows a general cross-sectional schematic of an open channel, in which $\alpha$ is the angle between the solid surface and the $z$-direction, and $\beta$ is the angle between the channel wall and the $z$-direction at the top of the channel.

A change in $r$ alters the cross-sectional area of the open channel, thus the effective flow resistance of the open channel also changes. Surface tension prevents the open portion of the channel from flooding, although this does occur under certain $P_{\mathrm{S}}: P_{\mathrm{D}}: P_{\mathrm{AIR}}$ relationships which will be discussed in the Robustness section.
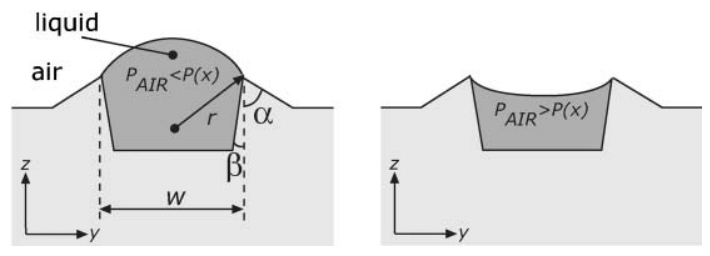

Fig. 2 Cross-section of channel with two different pressure differences across the open channel. The open liquid surface wicks up if $P(x)>P_{\mathrm{AIR}}$ (left) and wicks down if $P(x)<P_{\mathrm{AIR}}$ (right). Note that the cross sectional area is larger (thus the flow resistance lower) when the liquid wicks up rather than down.
The closed-open-closed flow component diminishes the impact of evaporation that is common in static open systems since only a small part of the liquid is exposed to the air compared with fully open systems. This component allows all the possibilities available with closed microfluidic functionality (i.e., liquid control, filtering, separation, etc.) to be integrated upstream or downstream of the open channel section, while taking advantage of direct access to liquid in the open section.

\section{Theoretical flow regimes}

To characterize and test the performance of the closed-openclosed microchannel, a demonstrator component was fabricated. Fig. 3 shows a schematic of the device, consisting of a glass substrate (channel floor) and cut PDMS film (channel walls). The closed inlet and outlet channels were made of $0.25 \mathrm{~mm}$ inner diameter steel syringe tubes glued to the sandwich structure. The transparent materials constituting the demonstrator make photographs unsuitable for illustrating the device and its functionality, hence we use schematic drawings throughout this paper to illustrate functionality more clearly. The open channel section length, width, and height are $13 \mathrm{~mm}, 600 \mu \mathrm{m}$, and $100 \mu \mathrm{m}$, respectively. Hydrostatic liquid pressure was applied at the inlet and outlet and the liquid profile was monitored through a microscope (from above and from the side of the channel lengthwise).

Assuming the Weber number $W e=\left(\rho w u^{2}\right) / \gamma \ll 1$, inertial effects such as kinematic pressure changes are negligible. The pressure drop $P_{\mathrm{S}}-P(x)$ along the channel position $x$ is due to viscous shear stress on the solid-liquid surfaces and results in different cross-sectional liquid profiles along the length of the channel.

Fig. 4 shows a cross-sectional lengthwise view of the closedopen-closed microchannel assuming $P_{\mathrm{D}}$ is varied and $P_{\mathrm{S}}$ and $P_{\text {AIR }}$ are kept constant with $P_{\mathrm{S}}>P_{\text {AIR }}$. When $P_{\mathrm{D}}=P_{\mathrm{S}}$, the open liquid level is flat and there is no net flow of liquid from

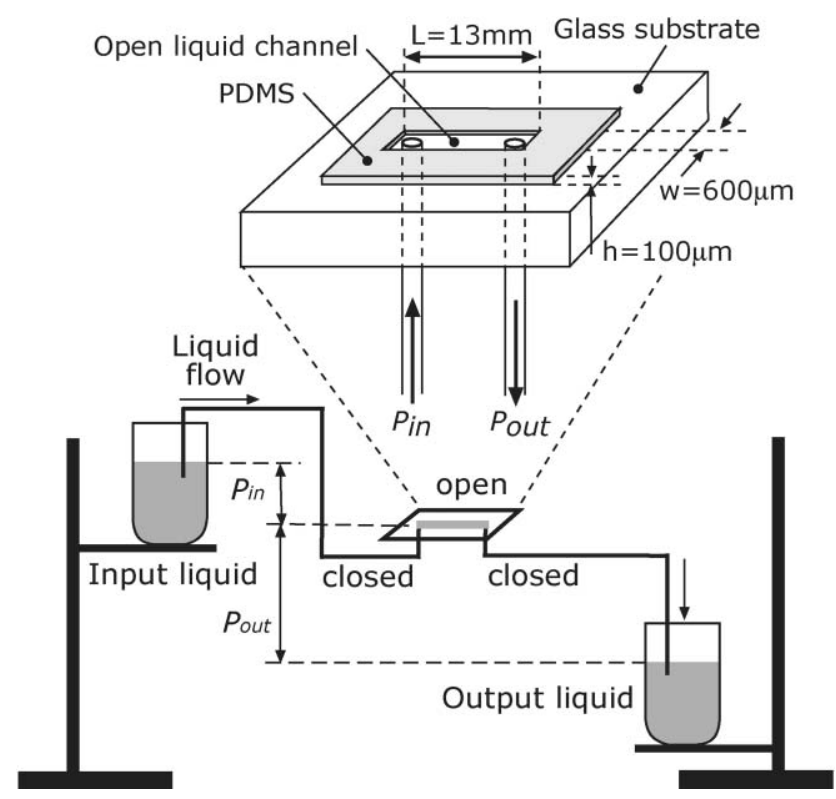

Fig. 3 Schematic of closed-open-closed microchannel demonstrator and measurement set-up. Coloured and uncoloured water was used. 


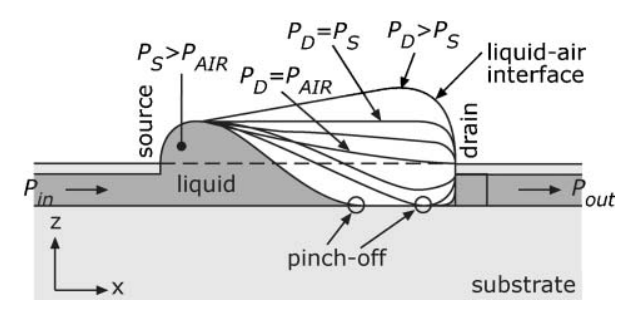

Fig. 4 Schematic representation of the closed-open-closed microchannel lengthwise cross-section. The dotted lines represent the liquid level under various pressure situations (varying $P_{\mathrm{D}}$ and keeping $P_{\mathrm{S}}$ and $P_{\text {AIR }}$ constant).

source to drain. When $P_{\mathrm{D}}>P_{\mathrm{S}}$, liquid flows from drain to source and the liquid height is greater at the drain compared with the source. If $P_{\mathrm{D}}$ is decreased to $P_{\mathrm{AIR}}$, liquid flows from source to drain and the liquid level slopes from source to drain. When $P_{\mathrm{D}}$ is decreased until the liquid level at the drain falls to the bottom of the channel, a dry spot is formed close to the drain. We call this situation 'pinch-off', and the critical liquid pressure at the pinch-off point is $P_{\mathrm{PO}}$. The flow resistance in the pinched channel section is dramatically increased. If $P_{\mathrm{D}}$ is decreased even further, the device behaviour will depend on the specific cross-sectional channel geometry. In our demonstrator, with a rectangular channel cross-section, liquid continues to flow along the bottom corners of the channel downstream of the pinch-off point, as is seen in Fig. 4. The dry region recedes lengthwise up the channel closer to the source if $P_{\mathrm{D}}$ is decreased. For other channel geometries, the very small flow might become unstable in this regime.

When we further investigated the cross-sectional behaviour of the microchannel, this time in a cross-sectional area perpendicular to the flow, we observed behaviour which is summarized in Fig. 5.† Referring to Fig. 5, when $P(x)=P_{\text {AIR }}$ the liquid level is approximately flush with the top surface of the microchannel. As $P(x)$ increases, the liquid surface wicks up until flooding occurs at a threshold pressure of $P_{\mathrm{FL}}$. If $P(x)$ falls below $P_{\mathrm{AIR}}$, the liquid surface wicks downwards until a certain value $P(x)=P_{\mathrm{DET}}$ where the air-liquid-solid 3-phase line detaches from the channel edge and moves towards the bottom of the channel. The detachment pressure, $P_{\mathrm{DET}}$, can be theoretically calculated as

$$
\begin{gathered}
P_{\mathrm{DET}}=-\frac{2 \gamma}{w} \sin \left(\beta+\theta_{\mathrm{c}}\right) \quad \text { for } \beta>-\theta_{\mathrm{c}} \\
P_{\mathrm{DET}}=-\frac{2 \gamma}{w_{c}} \quad \text { for } \beta>-\theta_{\mathrm{c}}
\end{gathered}
$$

in which $\theta_{\mathrm{c}}$ is the contact angle between the liquid and the solid surface. The flow resistance in the detached channel region is increased. The exact position of the 3-phase line during detachment, and whether detachment or pinch-off occur first when $P_{\mathrm{D}}$ is decreased, depend entirely on the specific channel cross-section.

$\uparrow$ The calculations of $P_{\mathrm{FL}}$ and $P_{\mathrm{DET}}$ are based on the following considerations: $^{9}$ the internal liquid pressure $P$ can be expressed as $P=-\mathrm{d} U_{\mathrm{T}} / \mathrm{d} V_{\mathrm{l}}$, in which $V_{\mathrm{l}}$ is the liquid volume and $U_{\mathrm{T}}=\gamma_{\mathrm{sa}} A_{\mathrm{sa}}+$ $\gamma_{\mathrm{sl}} A_{\mathrm{sl}}+\gamma_{\mathrm{la}} A_{\mathrm{la}}$ is the total interfacial energy of the system; the solid area $A_{\mathrm{sa}}+A_{\mathrm{sl}}$ is constant; and Young's equation $\gamma_{\mathrm{sa}}=\gamma_{\mathrm{sl}}+\gamma_{\mathrm{la}} \cos \theta_{\mathrm{c}}$. The system will strive to a minimum energy $U_{\mathrm{T}}$, i.e., a cylindrical shaped liquid-air interface.

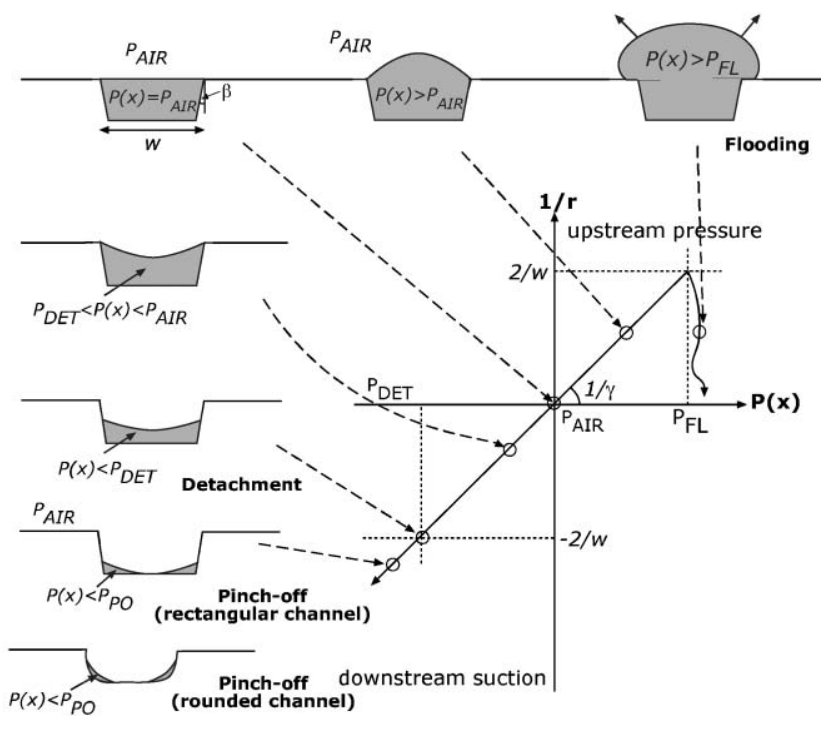

Fig. 5 Liquid curvature versus liquid pressure in the open channel.

\section{Robustness}

The two main possible failure modes for the closed-openclosed microchannel are (1) flooding and (2) bubbles sucked in at the source or drain. Flooding can occur during large upstream overpressures and is initiated at the source. When the internal liquid pressure $P(x)$ rises, the liquid wicks up, thus decreasing its radius of curvature. When the liquid pressure reaches the critical value $P_{\mathrm{FL}}$, further wicking of the liquid now increases the radius of curvature. The system cannot be in equilibrium at this point, and the channel floods. At this point, the liquid's radius of curvature is forced beyond its minimum and the top surface of the channel becomes wet. To reduce the risk of flooding, the angle $\alpha$ of the channel's upper edge can be designed such that more pressure can be tolerated. The critical internal liquid pressure, $P_{\mathrm{FL}}$, at which flooding is initiated can be calculated as

$$
\begin{gathered}
P_{\mathrm{FL}}=\frac{2 \gamma}{w} \sin \left(\frac{\pi}{2}+\theta_{\mathrm{c}}-\alpha\right) \text { for } \alpha>\theta_{\mathrm{c}} \\
P_{\mathrm{FL}}=\frac{2 \gamma}{w} \quad \text { for } \alpha>\theta_{\mathrm{c}} .
\end{gathered}
$$

To minimize the flooding risk, $\alpha$ should be less than $\theta_{\mathrm{c}}$ and the channel width, $w$, should be small. The top of the open channel should ideally be a highly hydrophobic and stable surface. Fig. 6 shows how a large angle $\alpha$ induces flooding in the open channel section at a lower liquid pressure compared to a small $\alpha$.

When large bubbles are present upstream of the open channel, the bubbles disappear into the surrounding air as they enter the open channel. Hence, this component acts as a debubbler to a certain extent. However, when $P_{\mathrm{D}}$ fell much below the pinch-off pressure, air bubbles were seen to be sucked into the drain. To reduce the risk of small bubbles entering the channel system via the open channel, the drain may be geometrically designed such that large capillary forces are present, as shown in Fig. 1. In this way, potential air bubbles experience a large counteracting capillary force when 


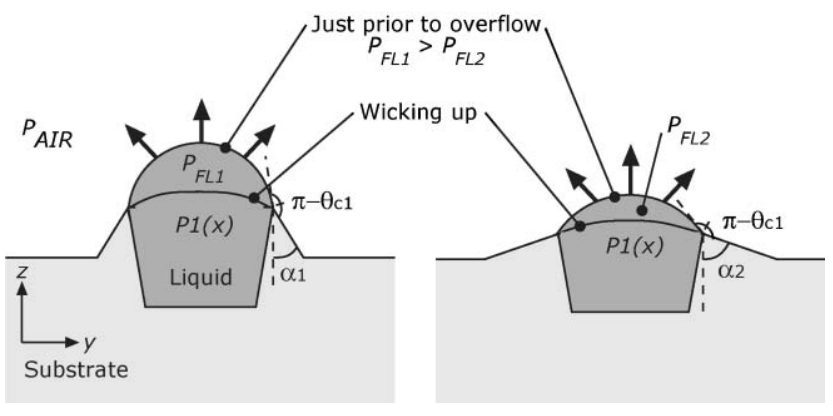

Fig. 6 Schematic cross-section of channel showing the effect of $\alpha$ and $\theta_{\mathrm{C}}$ on device flooding. Note that the liquid contact angle $\theta_{\mathrm{C} 1}$ is the same in both cases, but $\alpha_{2}>\alpha_{1}$. The left schematic shows an optimal geometry and the right schematic shows a suboptimal geometry.

entering the geometrical filter. The suction required to pull the air bubble through the filter is therefore very high.

The hydrophobic and hydrophilic nature of the materials used in the closed-open-closed microchannel are quite important for proper performance. After re-using a demonstrator device which had experienced flooding, the risk for subsequent floodings increased considerably. Depending on the channel geometry and surface material, either capillary priming occurs or pressure driven filling is required. Any small amount of evaporation occuring in the open channel section did not observably impair device functionality.

\section{Flow-pressure performance}

The flow-pressure performance of the closed-open-closed microchannel demonstrator was tested by varying the hydrostatic pressures $P_{\mathrm{IN}}$ and $P_{\mathrm{OUT}}$, see Fig. 3 . The flow rate was measured by weighing the amount of liquid exiting the outlet during a specific time interval. The theoretical flow-pressure characteristics of the demonstrator are modelled using HagenPoiseuille. ${ }^{10}$ For the cylindrical inlet and outlet channels, no-slip conditions were assumed at the cylindrical wall, while for the flow in the open channel section no-slip conditions were assumed at the channel bottom only (approximation justified by the large $w / h_{0}$ ratio). The flow resistance in the inlet and outlet tubes is expressed as

$$
R_{\mathrm{IN}}=R_{\mathrm{OUT}}=\frac{8 \mu L_{\mathrm{t}}}{w r_{\mathrm{t}}^{4}}
$$

where $\mu$ is the liquid viscosity, $r_{\mathrm{t}}$ is the syringe tube radius, and $L_{\mathrm{t}}$ is the tube length. The flow resistance of the open section in the channel is

$$
R_{\mathrm{OPEN}}=\frac{3 \mu L}{w h^{3}}
$$

where $L$ is the length of the open channel section, $w$ is the width, and $h$ is the liquid level height in the open section. The model of the open channel further assumed a constant liquid level, $h$, approximated by the average between the channel height, $h_{0}$, and the expected liquid level at the center of the channel, $h_{\mathrm{c}}$,

$$
h=\frac{h_{0}+h_{\mathrm{c}}}{2} .
$$

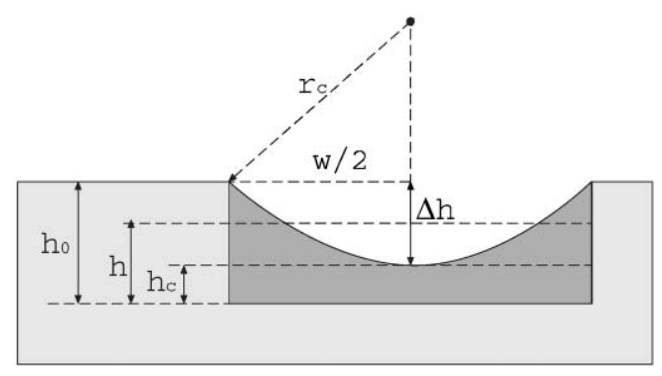

Fig. 7 Schematic showing parameter definitions for open channel model (cross-sectional view).

$h_{\mathrm{c}}$ can be calculated from the expected liquid pressure, $P_{\mathrm{c}}=P(L / 2)$, and liquid surface radius $r_{\mathrm{c}}$ at the channel centre (see Fig. 7):

$$
\begin{gathered}
P_{\mathrm{c}}=\frac{P_{\mathrm{S}}+P_{\mathrm{D}}}{2}=\frac{P_{\mathrm{IN}}+P_{\mathrm{OUT}}}{2} \\
r_{\mathrm{c}}=\frac{\gamma}{P_{\mathrm{c}}} \\
\Delta h=r_{\mathrm{c}}-\sqrt{r_{\mathrm{c}}^{2}-\left(\frac{w}{2}\right)^{2}} \\
h_{\mathrm{c}}=h_{0}+\frac{\Delta h}{2}
\end{gathered}
$$

The pinch-off and detachment flow regimes where not modelled since such a model depends entirely on the specific channel cross-sectional shape, making a geometry-general analysis impossible. Finally, the flow was calculated and plotted according to

$$
Q=\frac{P_{\mathrm{IN}}-P_{\mathrm{OUT}}}{R_{\mathrm{IN}}+R_{\mathrm{OUT}}+R_{\mathrm{OPEN}}} .
$$

Fig. 8 shows the measured and theoretical flow versus $P_{\mathrm{IN}}$ for $P_{\text {OUT }}=10 \mathrm{~Pa}$. As expected, pinch-off was first observed at

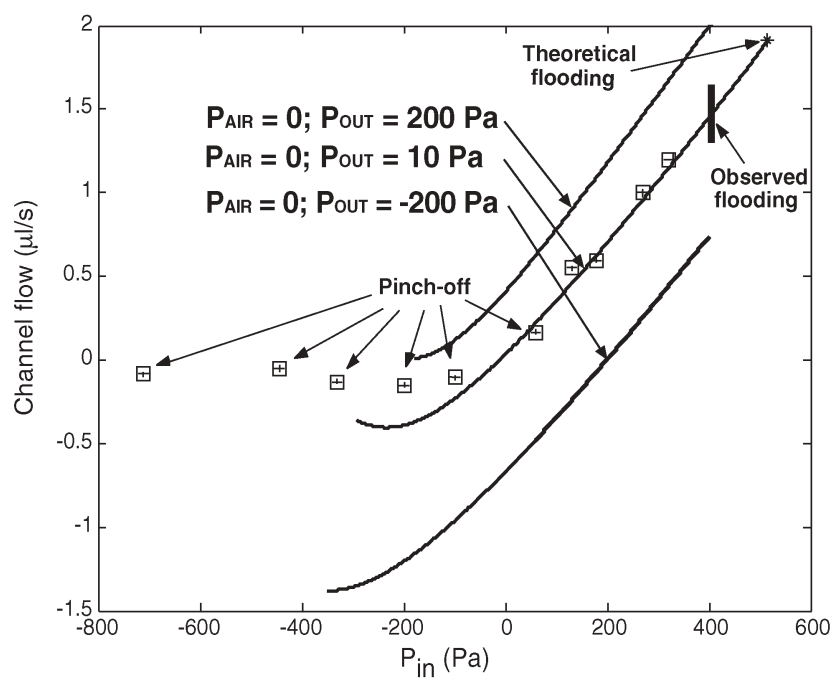

Fig. 8 Measured values (squares with error bars) and theoretical model (solid curves) of the inlet pressure $P_{\mathrm{IN}}$ versus flow in the closedopen-closed microchannel demonstrator. 


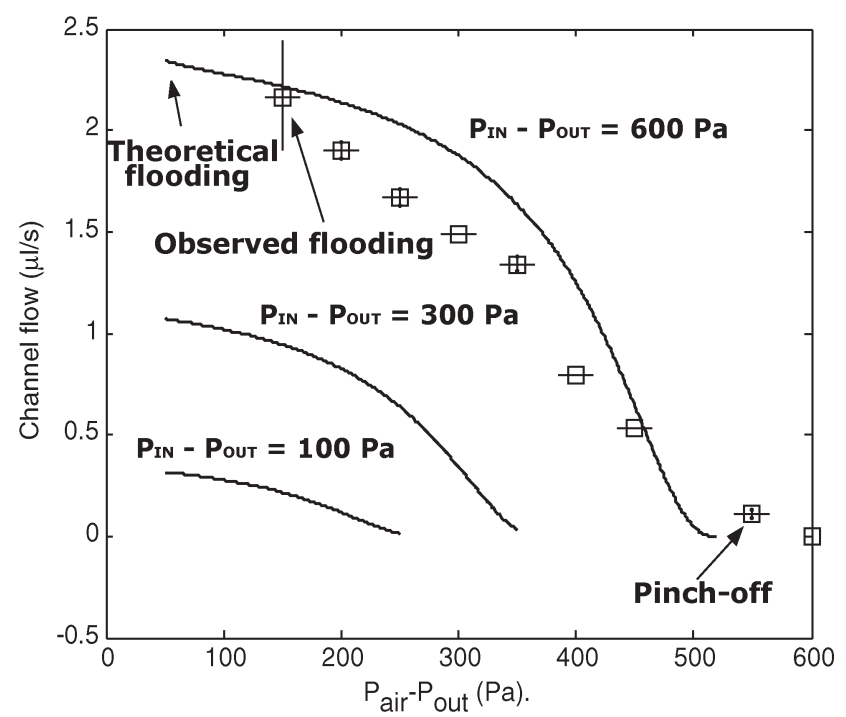

Fig. 9 Measured values (squares with error bars) and theoretical data (solid curves) of $P_{\mathrm{AIR}}-P_{\mathrm{OUT}}$ versus flow at various values of $P_{\mathrm{IN}}-$ $P_{\text {OUT }}$ using the microchannel demonstrator.

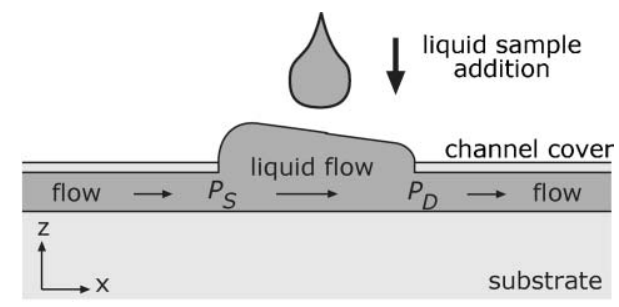

Fig. 10 Schematic of a droplet being pipetted into the open part of a closed-open-closed microchannel.

$P_{\mathrm{IN}} \approx P_{\text {OUT }}$. As $P_{\mathrm{IN}}$ was further decreased, the pinch-off point receded and little or no flow could be measured at the outlet. A linear flow curve is evident at higher $P_{\text {IN }}$ values where the flow resistance of the inlet and outlet channels dominates. This is the working region for the device if reliable continuous flow rates are desired. Flooding was observed slightly below the calculated value, but was likely caused by previous wetting of the top surface. Theoretical values matched measured values closely.

Fig. 9 shows the liquid flow in the channel while changing $P_{\text {AIR }}-P_{\text {OUT }}$ and keeping $P_{\text {IN }}$ constant. This was achieved by keeping the input and output beakers stationary while successively changing the height of the closed-open-closed demontrator itself. Again, theoretical data corresponded well to the measurements.

\section{Microfluidic interfacing}

Generally, closed microfluidic systems utilize some type of tubing for addition of liquid samples. A variety of such solutions exist, ${ }^{11}$ but most are plagued by problems including large on-chip footprints, non-batch fabrication, dead volume, and bubble trapping during priming. ${ }^{12}$ Other critical considerations include mechanical strength, pressure drop, and leak tightness. The closed-open-closed system can be used for direct fluidic addition of liquid to a microfluidic system. This method does not require extra sample tube interconnecting (other than the inlet and outlet). Discrete samples of liquid were successfully pipetted into the open section of the component, see Fig. 10.

Within seconds after addition, the liquid sample was taken up by the channel system. The speed of sample uptake depends upon the volume of liquid added and the flow rate of liquid in the open channel. Care should be taken not to deteriorate the flooding robustness due to wetting of the upper surface of the substrate when adding liquid samples. An entire microfluidic system could be designed with multiple directly addressable liquid access sites using the closed-open-closed microchannel method.

\section{Conclusion}

The closed-open-closed microchannel possesses the versatility of upstream and downstream closed microfluidics along with open fluidic direct access. Care must be taken when designing the closed-open-closed microchannel in terms of geometry and material selection for robust performance. The closedopen-closed channel has interesting cross-sectional fluidic behaviour and flow characteristics. The channel may be operated most reliably in the 'non-pinch-off regime' where linear or near linear pressure-flow curves can be achieved. The device has the advantage of eliminating gas bubbles that were present upstream when these enter the open channel section. The unique fluidic behaviour of this device opens the door to a number of applications, including direct liquid sample interfacing, without the need for additional and bulky sample tubing.

Jessica Melin, Wouter van der Wijngaart* and Göran Stemme Royal Institute of Technology, Department of Signals, Sensors and Systems, Microsystem Technology, Osquldas väg 10, SE 10044 Stockholm, Sweden. E-mail: wouter@s3.kth.se; Fax: +468100858

\section{References}

$1 \mathrm{http}: / /$ www.nanogen.com

2 B. Zhao, J. S. Moore and D. J. Beebe, Science, 2001, 291, 1023.

3 K. Ichimura, S. K. Oh and M. Nakagawa, Science, 2000, 288, 1624.

4 G. J. M. Bruin, Electrophoresis, 2000, 21, 3931.

5 E. Litborn and J. Roeraade, J. Chromatogr., 2000, 745, 137.

6 W. van der Wijngaart, Designing microfluidic control components, $\mathrm{PhD}$ thesis, 2002.

7 J. Melin, W. van der Wijngaart and G. Stemme, $\mu$ TAS '04, Malmö, Sweden, 2004, vol. 2, p. 139.

8 F. U. Gast and H. Fiehn, Lab Chip, 2003, 3, 6N.

9 P. F. Man, C. H. Mastrangelo, M. A. Burns and D. T. Burke, MEMS '98, Heidelberg, Germany, p. 45.

10 F. M. White, Fluid Mechanics, McGraw-Hill, New York, 1979.

11 B. L. Gray, D. Jaeggi, N. J. Mourlas, B. P. van Drieenhuizen, K. R. Williams, N. I. Maluf and G. T. A. Kovacs, Sens. Actuators A, 1999, 77, 57.

12 J. Melin, N. Roxhed, G. Gimenez, P. Griss, W. van der Wijngaart and G. Stemme, Sens. Actuators B, 2004, 100, 463. 